\title{
Visualization of Spirochetes by Labeling Membrane Proteins With Fluorescent Biarsenical Dyes
}

\author{
Chadwick Hillman ${ }^{1}$, Philip E. Stewart ${ }^{1}$, Martin Strnad ${ }^{1,2,3}$, Hunter Stone ${ }^{1}$, Tregei Starr ${ }^{1}$, \\ Aaron Carmody ${ }^{4}$, Tyler J. Evans ${ }^{1}$, Valentina Carracoi ${ }^{1}$, Jenny Wachter ${ }^{1}$ and \\ Patricia A. Rosa ${ }^{1 *}$ \\ ${ }^{1}$ Laboratory of Bacteriology, Rocky Mountain Laboratories, National Institute of Allergy and Infectious Diseases, National \\ Institutes of Health, Hamilton, MT, United States, ${ }^{2}$ Institute of Parasitology, Biology Centre of the Czech Academy of \\ Sciences, České Budějovice, Czechia, ${ }^{3}$ Faculty of Science, University of South Bohemia in České Budějovice, České \\ Budějovice, Czechia, ${ }^{4}$ Research Technologies Section, Rocky Mountain Laboratories, National Institute of Allergy and \\ Infectious Diseases, National Institutes of Health, Hamilton, MT, United States
}

OPEN ACCESS

Edited by:

Tao Lin

Baylor College of Medicine

United States

Reviewed by:

Janakiram Seshu,

University of Texas at San Antonio,

United States

Robert D. Gilmore,

Centers for Disease Control and

Prevention (CDC), United States

Mathieu Picardeau,

Institut Pasteur, France

*Correspondence:

Patricia A. Rosa

prosa@niaid.nih.gov

Specialty section

This article was submitted to Molecular Bacterial Pathogenesis,

a section of the journal

Frontiers in Cellular and Infection Microbiology

Received: 06 June 2019

Accepted: 24 July 2019

Published: 20 August 2019

Citation:

Hillman C, Stewart PE, Strnad M

Stone H, Starr T, Carmody A,

Evans TJ, Carracoi V, Wachter J and

Rosa PA (2019) Visualization of

Spirochetes by Labeling Membrane

Proteins With Fluorescent

Biarsenical Dyes.

Front. Cell. Infect. Microbiol. 9:287.

doi: 10.3389/fcimb.2019.00287
Numerous methods exist for fluorescently labeling proteins either as direct fusion proteins (GFP, RFP, YFP, etc. - attached to the protein of interest) or utilizing accessory proteins to produce fluorescence (SNAP-tag, CLIP-tag), but the significant increase in size that these accompanying proteins add may hinder or impede proper protein folding, cellular localization, or oligomerization. Fluorescently labeling proteins with biarsenical dyes, like FIAsH, circumvents this issue by using a short 6-amino acid tetracysteine motif that binds the membrane-permeable dye and allows visualization of living cells. Here, we report the successful adaptation of FIAsH dye for live-cell imaging of two genera of spirochetes, Leptospira and Borrelia, by labeling inner or outer membrane proteins tagged with tetracysteine motifs. Visualization of labeled spirochetes was possible by fluorescence microscopy and flow cytometry. A subsequent increase in fluorescent signal intensity, including prolonged detection, was achieved by concatenating two copies of the 6-amino acid motif. Overall, we demonstrate several positive attributes of the biarsenical dye system in that the technique is broadly applicable across spirochete genera, the tetracysteine motif is stably retained and does not interfere with protein function throughout the $B$. burgdorferi infectious cycle, and the membrane-permeable nature of the dyes permits fluorescent detection of proteins in different cellular locations without the need for fixation or permeabilization. Using this method, new avenues of investigation into spirochete morphology and motility, previously inaccessible with large fluorescent proteins, can now be explored.

Keywords: spirochetes, Borrelia, Leptospira, fluorescent protein, tetracysteine tag, biarsenical dye

\section{INTRODUCTION}

The phylum Spirochaetes contains multiple members of medical and veterinary concern, which include Borrelia species (Lyme disease and relapsing fever) and pathogenic Leptospira species (leptospirosis). Both genera exhibit distinctive morphologies with periplasmic flagella encompassed between inner and outer membranes, which, combined with their unique shape and structure, allow these pathogens to move quickly through tissues during infection. However, the molecular 
techniques available to identify virulence determinants and key cellular factors are rudimentary in spirochetes compared to those available for some members of the Enterobacteriaceae. Such limitations have hampered efforts to identify proteins involved in cell shape, spatial localization of proteins, and transport across the periplasmic space while accommodating flagellar rotation. To address some of the molecular mechanisms underpinning these and other cellular processes, fluorescent proteins and dyes have proven to be valuable tools, permitting detection of spirochetehost interactions (Moriarty et al., 2008; Norman et al., 2008; Dunham-Ems et al., 2009; Bockenstedt et al., 2012; Carrasco et al., 2015; Teixeira et al., 2016; Krishnavajhala et al., 2017), localization or transport of proteins within the bacterial cell (Schulze and Zuckert, 2006; Schulze et al., 2010; Xu et al., 2011; Zhang et al., 2015), or as reporter systems for gene expression (Carroll et al., 2003; Bykowski et al., 2006; Clifton et al., 2006; Eggers et al., 2006; Miller et al., 2006; Gautam et al., 2008, 2009; Whetstine et al., 2009; Aviat et al., 2010; Cerqueira et al., 2011; Grove et al., 2017; Matsunaga and Haake, 2018; Takacs et al., 2018). However, the use of fluorescent protein fusions, most commonly with green or red fluorescent protein (GFP or RFP, respectively), has limitations: the bulkiness of these proteins, typically $25-30 \mathrm{kDa}$, can lead to atypical protein localization and irregular cellular trafficking (Senf et al., 2008), and some FP alleles have specific $\mathrm{pH}$ or oxygen requirements that are not always compatible with the targeted location or underlying biological question. More recent adaptations, such as SNAP- and CLIPtags, are comparable in size $(20 \mathrm{kDA})$ and require an additional extraneous chemical agent that could impact cell growth and viability in order to fluoresce.

In an attempt to subvert size-related problems caused by fluorescent protein fusions, Griffin and Tsien developed a system for intra- and extra-cellular in vitro protein labeling using biarsenical dyes that bind specifically to tetracysteine motifs (Griffin et al., 1998). This method uses a synthetic fluorescent biarsenical compound, such as Fluorescein Arsenical Helix binder (FlAsH) or Resorufin Arsenical Helix binder (ReAsH), which forms a stable complex with a tetracysteine motif consisting of six amino acids (CCPGCC). The central two amino acids of the spacer (proline plus glycine) create a hairpin that reduces steric hindrance between the arsenical groups and the tetracysteine motif, yielding optimum fluorescence (Adams et al., 2002). Using this technique, fluorescent live cells can be viewed in real-time without the issues typically encountered with GFP and other accessory proteins.

Biarsenical dyes have been widely used to study protein dynamics and interactions in various bacteria, viruses and even prions. In an attempt to view components of the Type II secretion system in Pseudomonas, Senf et al. initially used GFP fused to their target protein, but the fusion resulted in a nonfunctional and unstable protein (Senf et al., 2008). In contrast, the nominal mass added by the tetracysteine motif did not perturb the system and allowed successful visualization of the Type II secretion system. Likewise, groups studying viral kinetics encountered similar obstacles with GFP-fusions of polyproteins, which disrupted normal viral function (Panchal et al., 2003; Arhel et al., 2006). However, both groups successfully employed the much smaller tetracysteine tags coupled with biarsenical dyes to visualize HIV infection and Ebola virus assembly. Other examples of the successful use of tetracysteine tags include the visualization of flagellar dynamics in E. coli communities (Copeland et al., 2010), Shigella effector components of the Type III secretion system entering host cells in real time (Enninga et al., 2005), and converted forms of prion proteins (Gaspersic et al., 2010). More recently, biarsenical dyes have been used to gain an understanding of flagellar elongation and decay in E. coli (Zhao et al., 2018). The broad application potential of tetracysteine motifs coupled with biarsenical dyes provides an alternative fluorescent method to visualize cells and proteins when fusion to larger fluorescent proteins may produce aberrant results.

Here, our group utilized tetracysteine motifs and biarsenical dyes to successfully label membrane proteins in live cells of the spirochetes Leptospira biflexa and Borrelia burgdorferi. We found that the biarsenical dyes can diffuse across the outer membranes of these spirochetes, and that concatenating two copies of the 6-amino acid tag increased the intensity and duration of fluorescence. Mouse-tick infection studies of a tetracysteine-tagged B. burgdorferi strain demonstrated that the tetracysteine motif was stably maintained in vivo and did not adversely affect infectivity in either the arthropod vector or murine host. Finally, biarsenical-bound proteins could be followed over several days, indicating that this approach can be used in time-course studies of live cells.

\section{MATERIALS AND METHODS}

\section{Bacterial Strains and Growth Conditions}

All strains used in this study are listed in Table 1. Borrelia burgdorferi strain B31 A3 is an infectious clonal derivative of the type strain B31 (Burgdorfer et al., 1982; Elias et al., 2002). B. burgdorferi $\Delta \mathrm{D} 109$, a previously characterized B31 A3 mutant lacking a wild-type copy of the plasmid-borne gene encoding outer-surface protein (Osp) D, was utilized in this study (Stewart et al., 2008). In addition, strain B31 A34, a non-infectious and more readily transformed clone that lacks restriction modification systems (Jewett et al., 2007), was also used. Borrelia cultures were grown at $35^{\circ} \mathrm{C}$ in liquid Barbour-Stoenner-Kelly (BSK)-II medium supplemented with $6 \%$ rabbit serum (Pel Freez Biologicals, Rogers, AZ) or in solid BSK medium under 2.5\% $\mathrm{CO}_{2}$ (Samuels et al., 1994). Borrelia growth media were supplemented with antibiotics at the following concentrations when appropriate: gentamicin $(40 \mu \mathrm{g} / \mathrm{mL})$, kanamycin $(200 \mu \mathrm{g} / \mathrm{mL})$, and streptomycin $(50 \mu \mathrm{g} / \mathrm{mL})$.

Leptospira biflexa serovar Patoc (strain Patoc I) was cultured at $30^{\circ} \mathrm{C}$ in a shaking incubator at $150 \mathrm{RPM}$ in EMJH medium (Fisher Scientific). Solid EMJH media for plating included 1.2\% wt/vol Nobel agar (Fisher Scientific), and plates were inverted, sealed with parafilm and incubated at $30^{\circ} \mathrm{C}$ for up to 1 week. Leptospira growth media were supplemented with kanamycin $(20 \mu \mathrm{g} / \mathrm{mL})$ where appropriate.

Escherichia coli TOP10 cells (Invitrogen, Carlsbad, CA) were used for recombinant DNA cloning purposes unless 
TABLE 1 | Strains used in this study.

\begin{tabular}{|c|c|c|}
\hline Designation & Description & Purpose \\
\hline B. burgdorferi B31 A3 & Wild-type infectious (Elias et al., 2002) & Parental strain \\
\hline B. burgdorferi B31 A34 & Non-infectious/high-passage (Jewett et al., 2007) & Parental strain \\
\hline A34-SV-OspDTC & $\begin{array}{l}\text { OspD with a single tetracysteine tag expressed from shuttle vector, } \\
\text { B31 A34 background (this study). This strain produces both a tagged } \\
\text { and wild-type form of OspD }\end{array}$ & $\begin{array}{l}\text { Testing and optimization of FlAsH system in } \\
\text { B. burgdorferi }\end{array}$ \\
\hline A34-SV-OspD2xTC & $\begin{array}{l}\text { OspD with a double tetracysteine tag expressed from shuttle vector, } \\
\text { B31 A34 background (this study). This strain produces both a tagged } \\
\text { and wild-type form of OspD }\end{array}$ & $\begin{array}{l}\text { Evaluating the benefit of concatenating two } \\
\text { tetracysteine tags to increase fluorescence and } \\
\text { prolong detection }\end{array}$ \\
\hline A3 $\Delta$ OspD 109 & ospD deletion strain, A3 background (Stewart et al., 2008) & Parental strain lacking ospD \\
\hline$\Delta$ OspD-SV-TC & $\begin{array}{l}\text { OspD with a single tetracysteine tag expressed from shuttle vector, } \\
A 3 \Delta O \operatorname{spD} 109 \text { background (this study) }\end{array}$ & $\begin{array}{l}\text { Evaluation of fluorescence and duration of fluorescence } \\
\text { in a strain lacking a wild-type version of OspD }\end{array}$ \\
\hline$\Delta$ OspD-SV-2xTC & $\begin{array}{l}\text { OspD with a double tetracysteine tag expressed from shuttle vector, } \\
A 3 \Delta \text { OspD } 109 \text { background (this study) }\end{array}$ & $\begin{array}{l}\text { For comparing a double tetracysteine tag to a single } \\
\text { tag in a strain lacking a wild-type version of OspD; } \\
\text { used for OspD re-labeling experiments }\end{array}$ \\
\hline A3-LA7TC & $\begin{array}{l}\text { LA7 with a single tetracysteine tag created by allelic exchange with the } \\
\text { endogenous gene }\end{array}$ & $\begin{array}{l}\text { Used to assess effect of tetracysteine motif on a } \\
\text { protein that is important in the B. burgdorferi infectious } \\
\text { cycle }\end{array}$ \\
\hline $\begin{array}{l}\text { L. biflexa serovar patoc strain } \\
\text { Patoc I (Paris) }\end{array}$ & Wild-type & Parental strain \\
\hline OmpATC & $\begin{array}{l}\text { OmpA with a tetracysteine tag created by integration at the } \\
\text { endogenous locus (this study). This strain produces both a tagged } \\
\text { and wild-type form of OmpA }\end{array}$ & Testing FIAsH system in Leptospira \\
\hline
\end{tabular}

otherwise noted. Final antibiotic concentrations were as follows: gentamicin $(5 \mu \mathrm{g} / \mathrm{mL})$, kanamycin $(50 \mu \mathrm{g} / \mathrm{mL})$, and spectinomycin $(100 \mu \mathrm{g} / \mathrm{mL})$.

\section{Mutant Construction and Transformation}

Oligonucleotides used in mutant construction are listed in Table 2. All plasmids generated in this study were confirmed by sequencing and are listed in Table 3. A tetracysteine motif was added to the $3^{\prime}$ end of a second copy of ompA (Outer Membrane Protein A, UniProt accession \#: B0SQ62) in $L$. biflexa by targeted integration of a non-replicating plasmid at the endogenous locus. First, the primer pair A/B was used to amplify the ompA-like coding sequence and clone it into pGem-T EZ (Promega Inc., Madison, WI). Next, an inverse PCR reaction was used in conjunction with primer pair $C / D$ to add the tetracysteine tag (TC-tag) at the $3^{\prime}$ end of ompA, which results in an in-frame addition of the motif to the carboxy-terminus of OmpA. Amplicons were digested, selfligated, and transformed into E. coli Top 10 cells. Next, a kanamycin resistance cassette suitable for use in $L$. biflexa ( $\operatorname{lgBp} a p h 1)$ was cloned into the construct using the available XhoI restriction enzyme site. Lastly, the ampicillin resistance cassette within pGem-T EZ was inactivated by excising part of the coding region with the restriction enzyme AclI; the plasmid was subsequently self-ligated, transformed into E. coli Top 10 cells and selected on kanamycin plates. Colonies resistant to kanamycin were replica-plated for susceptibility to carbenicillin to demonstrate inactivation of the ampicillin resistance cassette. The completed targeted integration construct, pGem::OmpA-TC, was transformed into L. biflexa using protocols previously established (Louvel and Picardeau, 2007) and the resulting strain designated OmpATC. Transformants were confirmed by PCR (Figure 1A) and Southern blot analysis (data not shown).

The TC-tag was incorporated into the $3^{\prime}$ end of the $o s p D$ coding sequence by PCR amplification using primers $\mathrm{G}$ and $\mathrm{H}$, to create ospDTC. This amplicon includes the putative promoter $5^{\prime}$ of the ospD gene (Stewart et al., 2008) and $15 \mathrm{bp}$ homologous to the multiple cloning site of shuttle vector pKFSS1 (Frank et al., 2003) The resulting PCR product was cloned into pKFSS1 using the In-fusion kit (Takara, Mountain View, CA), following the manufacturer's recommendations, and transformed into E. coli Stellar cells.

The ospD-2xTC cassette was cloned into the B. burgdorferi shuttle vector pBSV2G (Elias et al., 2003). An amino acid pocket consisting of GDEG was placed between the dual tetracysteine motifs, as described previously (Andresen et al., 2004). Briefly, primer pair I/J was used to PCR- amplify OspD-1xTC and add a second tetracysteine motif at the $3^{\prime}$ end of the PCR amplicon with primer J. PCR products were digested with SalI and ligated into pBSV2G to form the shuttle vector pBSV2G::OspD2xTC.

The coding region of la7 was replaced with la7-TC by allelic replacement using a construct encoding TC-tagged LA7 cloned into pGEM-T EZ. Briefly, primers $\mathrm{L}$ and $\mathrm{M}$ were used to PCR-amplify la7, including $\sim 500$ bp upstream and downstream, of B. burgdorferi and clone into pGEM-T EZ. Next, the primer pair $\mathrm{N} / \mathrm{O}$ was used in an inverse PCR reaction to add the tetracysteine motif to the $3^{\prime}$ end of la7. Amplicons were digested, self-ligated, and transformed into E. coli Top 10 cells. A kanamycin resistance cassette suitable for use in 
TABLE 2 | Primers used in this study.

Primers

\begin{tabular}{|c|c|c|c|}
\hline & Name & Sequence & Function \\
\hline \multicolumn{4}{|c|}{ LEPTOSPIRA } \\
\hline A & OmpA.SnaBI.For & tacgtaGAGCAG TCCGTTGACAAG & Cloning of ompA \\
\hline B & OmpA.SnaBI.Rev & tacgtaTCG TCTGGTAAGGAT TGG & Cloning of ompA \\
\hline C & iPCR.OmpA.Xhol.For & ctcgagGAAATTCTATITCTTACTAGAGACC & Inverse PCR for addition of tetracysteine motif \\
\hline D & $\begin{array}{l}\text { iPCR.OmpA.TC. } \\
\text { Xhol.Rev }\end{array}$ & $\begin{array}{l}\text { ctcgagTTAACAACATCCAGGGCAACATTAGAAACGA } \\
\text { CTTGGAAAGTCAC }\end{array}$ & $\begin{array}{l}\text { Genetically encoding the tetracysteine motif at the } 3^{\prime} \text { end } \\
\text { of ompA before the stop codon (underlined) }\end{array}$ \\
\hline $\mathrm{F}$ & Kan.Out.RC & GCAGTTCATTTGATGCTCG & Transformant confirmation by PCR \\
\hline \multicolumn{4}{|c|}{ BORRELIA } \\
\hline & OspD1xTC & & \\
\hline G & PospD-TC.SV2.F & CGGTACCCGGGGATCGGCCATGGGAAGAAGGAG & Amplifying $o s p D$ and cloning into pKFSSI \\
\hline $\mathrm{H}$ & OspD-TC.SV2.RC & $\begin{array}{l}\text { ATGCCTGCAGGTCGATTAACAACATCCAGGGCAAC } \\
\text { AAGTATTTAACAAGGCCACAACTTC }\end{array}$ & $\begin{array}{l}\text { Genetically encoding the tetracysteine motif at the } 3^{\prime} \text { end } \\
\text { of ospD before the stop codon (underlined) }\end{array}$ \\
\hline \multirow[t]{2}{*}{ K } & OspD.For & GCTCTCAATATCTTGTGTTC & Transformant confirmation by PCR \\
\hline & LA7TC & & \\
\hline L & LA7.SnaB1.For & tacgtaGCATCAAGTCTTGGTGAATCTG & Cloning of la7 \\
\hline M & LA7.SnaB1.Rev & tacgtaCTAGAAATAGACTATGGGCAAGG & Cloning of la7 \\
\hline $\mathrm{N}$ & $\begin{array}{l}\text { iPCR.LA7.Xhol. } \\
\text { For }\end{array}$ & TATTctcgag TTATATIITGATTTATAGGCTITAATC & Inverse PCR for addition of tetracysteine motif \\
\hline $\mathrm{O}$ & iPCR.LA7.TC. Xhol.Rev & $\begin{array}{l}\text { ATctcgagTTAACAACATCCAGGGCAACAATTCG } \\
\text { TTAACATAGGTGAAATIIITCAACG }\end{array}$ & $\begin{array}{l}\text { Genetically encoding the tetracysteine motif at the } 3^{\prime} \text { end } \\
\text { of la7 before the stop codon (underlined) }\end{array}$ \\
\hline$P$ & LA7.Seq3. For & GCACGTITTCACGCTATG & Transformant confirmation by PCR \\
\hline Q & Kan736.RC & AAAGCCGTTTCTGTAATGAAGGAG & Transformant confirmation by PCR \\
\hline
\end{tabular}

All oligos are shown in the $5^{\prime}$ to $3^{\prime}$ orientation. Restriction enzyme sites are depicted in the primer name and sequences are indicated in lower case. Stop codon sequences are underlined where applicable.

TABLE 3 | Plasmids used in this study.

\begin{tabular}{|c|c|c|}
\hline & Function & References \\
\hline \multicolumn{3}{|l|}{ Plasmids } \\
\hline pGem-TEZ & $\begin{array}{l}\text { Backbone for ompA } \\
\text { integration construct }\end{array}$ & Promega \\
\hline pKFSS1 & $\begin{array}{l}\text { Shuttle vector for } \\
\text { B. burgdorferi }\end{array}$ & $\begin{array}{l}\text { Frank et al., } \\
2003\end{array}$ \\
\hline pBSV2G & $\begin{array}{l}\text { Shuttle vector for } \\
\text { B. burgdorferi }\end{array}$ & Elias et al., 2003 \\
\hline pGem::OmpA-TC & $\begin{array}{l}\text { OmpA-TC suicide vector for } \\
\text { L. biflexa }\end{array}$ & Current study \\
\hline pKFFS1::OspD1xTC & OspD-TC shuttle vector & Current study \\
\hline pBSV2G::OspD2xTC & OspD-2xTC shuttle vector & Current study \\
\hline pGEM::LA7-TC & $\begin{array}{l}\text { LA7-TC allelic exchange } \\
\text { vector for } B \text {. burgdorferi }\end{array}$ & Current study \\
\hline
\end{tabular}

B. burgdorferi (flgBp aph1) was added to the construct using the available XhoI restriction enzyme site introduced at the previous inverse PCR step. Lastly, ampicillin resistance was inactivated as described above to generate the completed allelic replacement construct, pGEM::LA7-TC.

Allelic exchange and shuttle vector constructs were transformed into B. burgdorferi by electroporation as previously described (Samuels et al., 1994). Strains A34-SV-OspDTC and A34-SV-OspD2xTC were created in the high-passaged, noninfectious strain B31 A34, which has a higher transformation frequency than the parental strain B31 A3 and also carries a wild-type copy of the ospD gene. Strains $\triangle$ OspD-SVTC and $\triangle$ OspD-SV-2xTC were produced by transforming pKFFS1::OspD1xTC or pBSV2G::OspD2xTC into A3 $\triangle \mathrm{D} 109$, a B31 A3 derivative lacking a wild-type copy of ospD (described by Stewart et al., 2008). Both OspD tetracysteine constructs are on shuttle vectors present at multiple copies per cell in Borrelia. Note that strain $\triangle \mathrm{OspD}-\mathrm{SV}-2 \mathrm{xTC}$ produces only an OspD-TC protein, whereas B31 A34-derived strains produce both wild-type OspD (lacking a TC tag) and TC-tagged OspD. In contrast, A3-LA7TC was constructed by an allelic exchange event that replaced the wild-type copy of LA7 on the chromosome in strain B31 A3. All strains were confirmed by restriction enzyme digestion, sequencing, PCR, and immunoblotting. Rabbit anti-OspD antiserum was used at dilution of 1:1,000 
and rabbit anti-LA7 antiserum (kindly provided by Dr. Brian Stevenson) at 1:250.

\section{FIAsH/ReAsH Assays}

Spirochetes were grown to mid-exponential phase $\left(\sim 5 \times 10^{7}\right.$ cells $/ \mathrm{mL}$ for $B$. burgdorferi and $\sim 5 \times 10^{8}$ cells $/ \mathrm{mL}$ for $L$. biflexa) and harvested by centrifugation (5,800 RCF for B. burgdorferi and 4,100 RCF for L. biflexa) for $10 \mathrm{~min}$ at room temperature. Pelleted spirochetes were then washed with $1 \mathrm{~mL}$ FlAsH wash buffer (50 mM MOPS pH 7.2, $67 \mathrm{mM} \mathrm{NaCl}, 20 \mathrm{mM} \mathrm{NH}_{4} \mathrm{Cl}$ ). Spirochetes were again pelleted and washed in FlAsH wash buffer with $20 \mathrm{mM}$ DTT. After the second wash, pelleted cells were resuspended in $158 \mu \mathrm{L}$ of FlAsH solution and incubated in the dark at room temperature for $1 \mathrm{~h}$. The FlAsH or ReAsH solution for staining spirochetes was prepared as follows: $2 \mathrm{mM}$

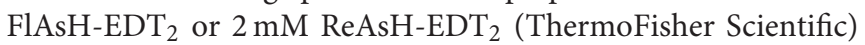
was resuspended in FlAsH wash buffer at a final concentration of $4.75 \mu \mathrm{M}$, and DTT was added to a final concentration of $2.5 \mathrm{mM}$. The solution was then passed through a $0.22 \mu \mathrm{M}$ filter, and $2 \mathrm{M}$ DTT was added at $1 / 100 \mathrm{v} / \mathrm{v}$ to a final concentration of $22.5 \mathrm{mM}$. After labeling, $1 \mathrm{~mL}$ of FlAsH wash buffer was added and cells were pelleted as described above. Lastly, spirochetes were resuspended in $\sim 50 \mu \mathrm{L}$ FlAsH wash buffer for visualization.

\section{Microscopy}

All microscopy was done with a Nikon E80i fluorescent microscope. Image files were obtained and analyzed using Nikon Elements version 4.2 and ImageJ software version 2.0.0-rc69/1.52i. FlAsH dye was observed in the FITC channel while ReAsH was observed in the Texas Red channel. When comparing peak fluorescence, identical settings for exposure and gain were used for all images. Peak fluorescence data were obtained by setting a constant exposure and gain between strains (typically this was an exposure of $1 \mathrm{~s}$ with a gain of 9.6x); however, if these settings oversaturated the image sensor, the exposure was lowered for all strains tested. Average peak fluorescence data were obtained by placing a region of interest (ROI) box within a spirochete along an area of uniform fluorescence. The peak fluorescence of that box was calculated with Nikon Elements software and that value represented the peak fluorescence intensity for that spirochete. Approximately 250 spirochetes per group, and 100 measurements of background, pooled from 3 independent biological replicates, were analyzed.

\section{Flow Cytometry}

To assess fluorescence intensity of a larger number of spirochetes, cultures were first FlAsH- or ReAsH-stained, incubated with Hoechst 33342 DNA stain $(20 \mu \mathrm{M})$ (Thermofisher Scientific) for $30 \mathrm{~min}$ at room temperature, and then analyzed with an LSR II BD Flow Cytometer. Spirochetes were gated based on forward scatter (FSC), side scatter (SSC), and FITC. B31 A34 was utilized as a non-fluorescent wild-type control in conjunction with Hoechst 33342 to identify the spirochete populations, while FITC was used to detect the fluorescent spirochete population. B31 A34-derived strains were utilized to avoid introduction of infectious material in the flow cytometer. A34-SV-OspDTC and A34-SV-OspD2xTC were used to determine the effect of concatenating tetracysteine motifs on OspD. FlowJo version 10.4.2 software was used to analyze data and to calculate geometric means of populations.

\section{Animal Studies}

Rocky Mountain Laboratories (RML) is accredited by the International Association for Assessment and Accreditation of Laboratory Animal Care. Protocols for animal experiments were prepared according to the guidelines of the National Institutes of Health and approved by the RML Animal Care and Use Committee. RML mice are an outbred colony of Swiss-Webster mice maintained at Rocky Mountain Laboratories and used exclusively throughout this study. Mice were inoculated with $\sim 5 \times 10^{3}$ spirochetes intraperitoneally and $1 \times 10^{3}$ spirochetes subcutaneously. Infection was assessed by attempted isolation of spirochetes from ear, bladder, and rear ankle joint tissues at 3 weeks post-inoculation.

Larval Ixodes scapularis were reared from egg masses laid by engorged female ticks purchased from Oklahoma State University. All ticks were maintained in a temperature- and humidity-controlled chamber (Caron Model $7000-25$ ) at $22^{\circ} \mathrm{C}$ with $95 \%$ relative humidity. Approximately 100 larvae or 10-20 nymphs per mouse were allowed to feed to repletion. A subset of fed nymphs was crushed and the resulting homogenate serially diluted and plated to determine spirochete burden. Fluorescence of FlAsH-stained organisms was assessed immediately for spirochetes derived from mechanically-disrupted, engorged nymph midguts, and from cultured spirochetes isolated from ticks and mouse tissues.

\section{Time-Course of OspD-Biarsenical Dye Fluorescence}

To assess the longevity of FlAsH fluorescence during in vitro cultivation, B. burgdorferi cells were FlAsH-labeled as described above. Strains lacking a tetracysteine tag were used as negative controls for the procedure. For heat-killed cells, samples were placed at $55^{\circ} \mathrm{C}$ for $15 \mathrm{~min}$ post-FlAsH labeling and observed subsequently for absence of motility; these cells served as a control for passive loss of fluorescence over time. Labeled cells were resuspended in 1-2 mL of BSK-H medium (Sigma) with Borrelia antibiotics $(20 \mu \mathrm{g} / \mathrm{ml}$ phosphomycin, $50 \mu \mathrm{g} / \mathrm{ml}$ rifampicin, $2.5 \mu \mathrm{g} / \mathrm{ml}$ amphotericin B), placed in cryovials (Corning), and incubated at $22^{\circ} \mathrm{C}$ in the dark. Spirochetes were counted using Petroff-Hauser chambers to monitor growth, and all cultures were imaged every $48 \mathrm{~h}$ for 7-8 days. Experiments were performed as 3 biological replicates and the images shown come from a single experiment representative of the trends observed.

Counter-labeling assays were performed in order to assess FlAsH saturation of OspD protein over time. In cells originally stained with FlAsH where cell concentrations and images had already been obtained for comparison, $400 \mu \mathrm{L}$ aliquots were removed, and a ReAsH assay performed as stated above.

\section{Statistics}

Statistical analyses were conducted using GraphPad Prism 7 software. Mann-Whitney statistical analysis was applied when 

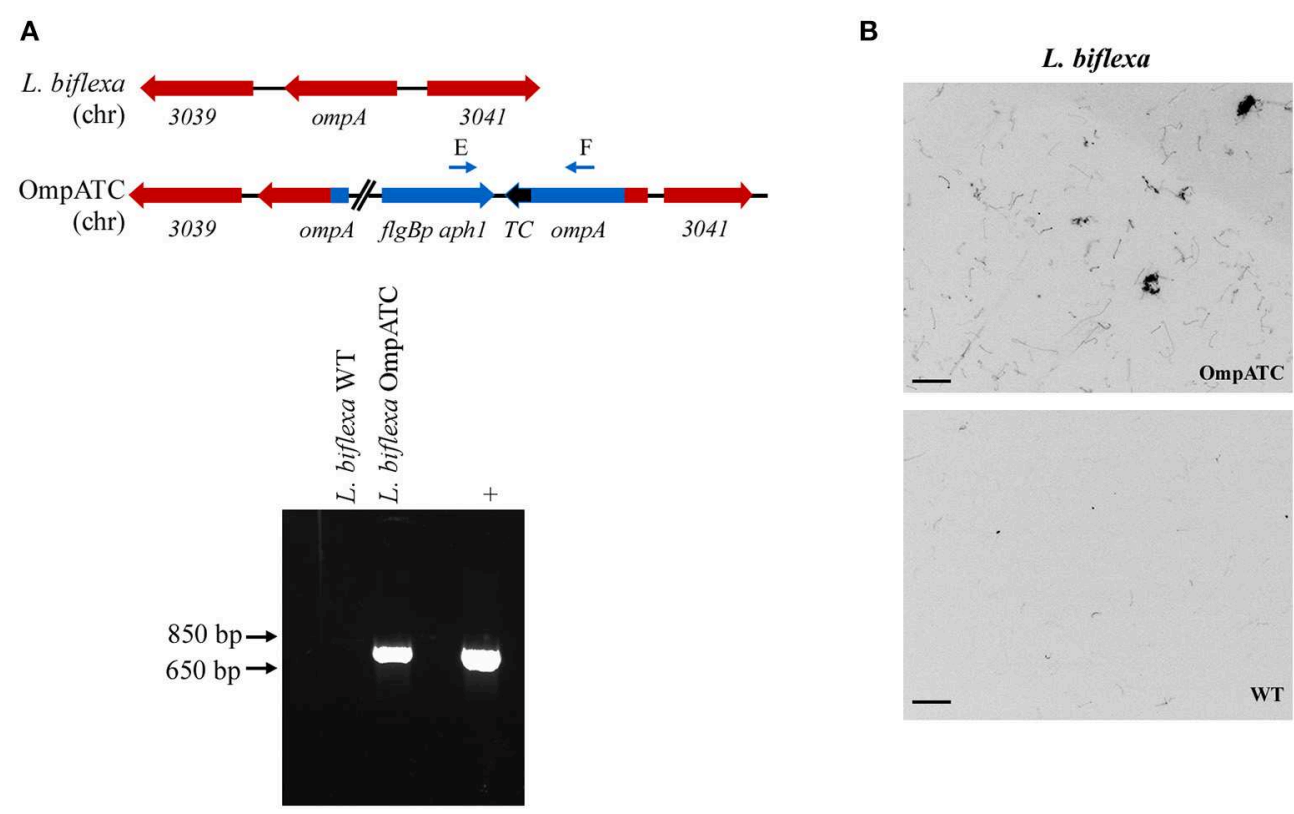

FIGURE 1 | Confirmation of genetically modified and fluorescent L. biflexa spirochetes by PCR and microscopy. (A) Diagram of relevant genetic loci and corresponding agarose gel image of PCR amplicons. The region in blue denotes the integrated plasmid DNA (plasmid sequences between ompA and aphl cassette not depicted in diagram), while endogenous loci are depicted in red. Small arrows above diagram indicate oligonucleotides $\mathrm{E}$ and $\mathrm{F}$ used for PCR confirmation of the ompATC integration. Transforming plasmid DNA serves as a positive control (+), while L. biflexa WT DNA serves as a negative control. (B) L. biflexa OmpA fluorescence. FIAsH dye-staining of the tetracysteine-tagged OmpATC strain and WT L. biflexa (negative control). Scale bar $10 \mu \mathrm{m}$; image inverted.

comparing fluorescence intensity by microscopy, geometric mean variation by flow cytometry, and spirochete burden in nymphs.

\section{RESULTS}

\section{Tetracysteine Tags and Biarsenical Dyes to Visualize Spirochetes}

As proof of principle, we assessed the use of biarsenical dyes in the model organism L. biflexa. Although membrane proteins have not been extensively characterized in this spirochete, a previous study identified a moderately abundant membrane protein, OmpA (Stewart et al., 2016). To assess the functionality of the FlAsH dye technique in Leptospira, a tetracysteine tag was added to the carboxy-terminus of OmpA encoded by a second copy of the gene integrated at the endogenous locus (Figure 1A). Transformants were confirmed by PCR utilizing a primer pair spanning the region between $\operatorname{omp} A$ and the kanamycin resistance cassette (Figure 1A), and transformed spirochetes fluoresced when exposed to FlAsH dye, permitting visualization of the helical shape characteristic of Leptospira cells (OmpATC in Figure 1B). In contrast, cells that did not contain a tetracysteine motif (WT negative control, Figure 1B) did not fluoresce when exposed to the biarsenical dye and only minimal background staining was observed.

To broaden the utility of this technique, we used a similar approach in another spirochete, B. burgdorferi, and tagged both outer and inner membrane proteins OspD and
LA7, respectively. These abundant and accessible lipoproteins should be suitable candidates for applying the FlAsH dye technique in B. burgdorferi. Spirochetes transformed with a shuttle vector encoding ospDTC were confirmed by PCR with primers specific to the $\operatorname{ssp} D$ coding region and the tetracysteine motif; wild-type cells that did not contain the motif did not yield an amplicon (Figure 2A, lower panel). Immunoblot analysis of OspD production in all engineered strains indicated an increase in OspD protein in strains transformed with a shuttle vector encoding either a single tetracysteine motif, A34SV-OspDTC, or two tetracysteine motifs, A34-SV-OspD2xT (Supplementary Figure 1A). LA7, originally proposed to be an outer membrane lipoprotein (Grewe and Nuske, 1996), but subsequently shown to localize primarily to the inner membrane (von Lackum et al., 2007; Yang et al., 2013), was chosen as a target to assess the ability of biarsenical dyes to freely diffuse across the outer membrane of living $B$. burgdorferi cells. Allelic exchange transformants were screened by PCR using a primer set spanning la7 and the kanamycin resistance cassette (Figure 2B, lower panel), and spirochetes containing a tetracysteine motif engineered on LA7 (strain A3LA7TC) produced comparable levels of protein as their wild-type counterpart (Supplementary Figure 1B).

After confirming the presence of the tetracysteine motif in all strains, we next assessed the FlAsH system in $B$. burgdorferi. Fluorescent spirochetes were observed when the tagged strains A34-SV-OspDTC and A3-LA7TC were exposed to FlAsH or ReAsH dye, whereas WT B31 A34 spirochetes (negative control) displayed only background fluorescence (Figure 3). 
A

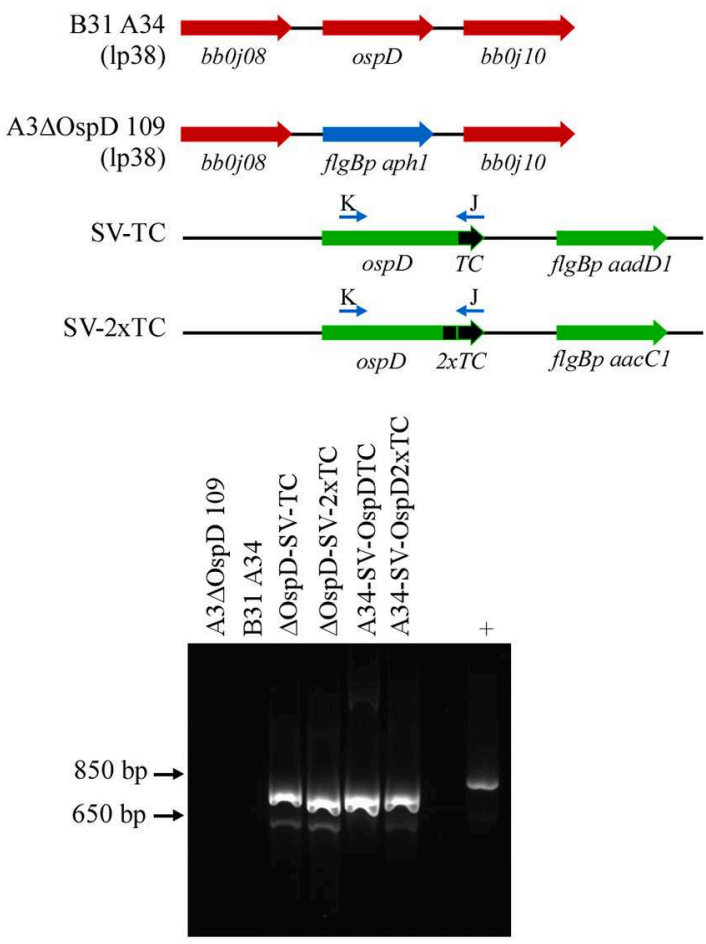

B

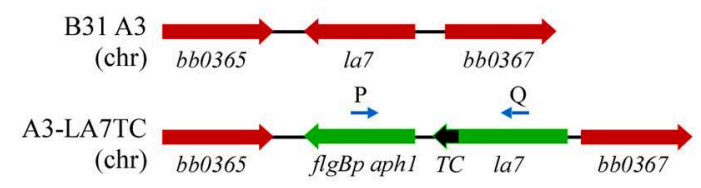

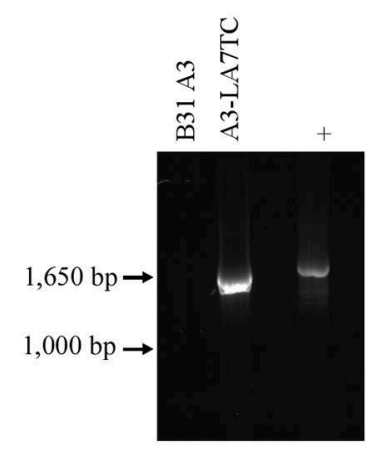

FIGURE 2 | PCR confirmation of genetically modified B. burgdorferi spirochetes. (A) Diagram of relevant genetic loci and corresponding agarose gel image of PCR amplicons. Native loci are shown in red, the kanamycin-resistance cassette (flgBp aph1) is in blue, and relevant portions of the shuttle vectors SV-TC and SV-2xTC are shown in green. Small arrows above diagram indicate oligonucleotides $\mathrm{K}$ and J used for PCR confirmation of the ospDTC gene in B. burgdorferi transformants. The endogenous plasmid (Ip38) locus in the parental B. burgdorferi strains (A3 $\triangle \mathrm{OspD} 109$ and B31A34) does not amplify, while the transforming shuttle vector DNA serves as a positive control (+). (B) PCR amplification of the chromosomal la7TC region utilizing primers $P$ and $Q$ (small arrows above diagram). Native loci are shown in red, while DNA introduced by allelic exchange, including the kanamycin-resistance cassette (flgBp aph1), is shown in green. The parental B. burgdorferi strain (B31 A3) serves as a negative control, while the transforming plasmid DNA acts as a positive control.

This demonstrated successful application of the technique to inner and outer membrane proteins of B. burgdorferi, and thus confirms that biarsenical dyes can diffuse across the outer membrane of live spirochetes without the need for fixation or permeabilization procedures.

\section{Optimization of the FIAsH Technique}

Having ensured that biarsenical dyes could be utilized in spirochetes, we next assessed whether adding a second tetracysteine tag would augment fluorescence. Using identical imaging conditions between B31 A34, A34-SV-OspDTC, and A34-SV-OspD2xTC, a significant increase in fluorescence intensity was observed with addition of a second tetracysteine motif onto OspD (Figures 4A,B), while the overall OspD protein level appeared similar between strains A34-SV-OspDTC and A34-SV-OspD2xTC (Supplementary Figures 1A-D), indicating the increase in fluorescence was a result of the second tetracysteine motif. FlAsH dye-labeled OspD2xTC spirochetes retained motility and grew comparably to unlabeled organisms when incubated at 35 degrees, confirming their viability and the lack of toxicity of the protocol. Also, the addition of a second tetracysteine motif allowed prolonged detection of the fluorescent signal (see Supplementary Figure 2).

FlAsH-labeled spirochetes were also analyzed by flow cytometry to quantify and compare fluorescence of a larger number of cells (Figure 4C). Using this method, we independently validated that two tetracysteine tags fused to OspD resulted in a significant increase in mean fluorescence relative to cells containing OspD with a single tag (Figure 4D). Hence the relative mean fluorescence intensity of spirochetes measured by both methods, fluorescence microscopy and flow cytometry, were in agreement for these B. burgdorferi strains.

Surprisingly, while optimizing the protocol, we found that FlAsH dye would non-specifically bind to heat-killed B. burgdorferi cells in the absence of tetracysteine motifs (Supplementary Figure 3). The nature of this non-specific interaction between the dye and spirochetes lacking an engineered tetracysteine pocket is not clear. However, this fluorescence requires the FlAsH dye and is only detected in the FITC channel, indicating that it does not reflect general autofluorescence of dead spirochetes. It should be noted that although non-specific staining of heat-killed spirochetes could 


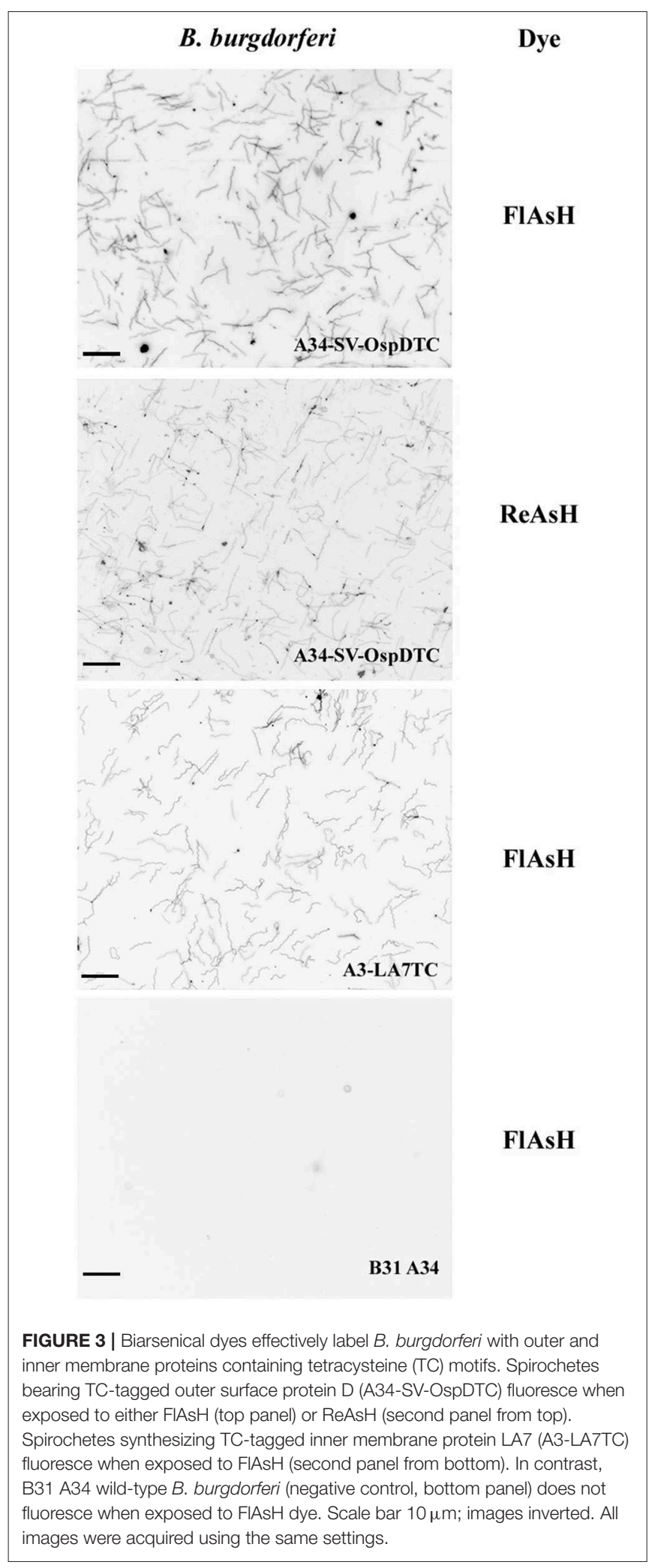

be abrogated by increasing the concentration of DTT, there was a concomitant decrease in the fluorescence intensity of viable spirochetes containing tetracysteine motifs (data not shown). Hence there is a balance to be achieved between the concentration of reducing agent and fluorescence intensity. Taking these observations into consideration, $20 \mathrm{mM}$ DTT was chosen as the optimal concentration, and WT spirochetes included as negative controls in all experiments to confirm specificity of labeling.

\section{Tetracysteine Motifs Are Stable Throughout the Mouse-Tick Infectious Cycle}

In nature, B. burgdorferi cycles between tick vectors and vertebrate hosts. Potentially, addition of the tetracysteine tag to a protein such as LA7, which is important during tick acquisition (Pal et al., 2008), might alter infectivity or destabilize the target transcript or protein in vivo. To examine this possibility, we needle-inoculated mice with either wild-type A3 or the A3LA7TC strain. All mice became infected ( 5 out of 5 mice for each strain), and both strains could subsequently be re-isolated from mouse tissues (ankle joint, bladder, and ear).

Before the A3-LA7TC-infected mice were euthanized for attempted spirochete isolation, they were fed upon by naïve $I$. scapularis larvae. A subset of replete larvae was mechanically disrupted and the resulting homogenates cultured to evaluate acquisition of B. burgdorferi and whether spirochetes retained the tetracysteine tag. Among larvae that fed on wild-type A3 or A3LA7TC-infected mice, 4/5 and 5/5 larvae, respectively, acquired spirochetes. Further, upon addition of FlAsH dye, all A3-LA7TC spirochetes isolated from larval ticks fluoresced (Figure 5A, topleft panel), indicating retention of the tetracysteine motif, while A3 spirochetes did not fluoresce (Supplementary Figure 4).

After molting to the nymphal stage, a subset of unfed ticks were mechanically disrupted and cultured to gauge the transstadial retention of $B$. burgdorferi. Of these, all of the A3LA7TC -infected nymphs (5 out of 5) and $80 \%$ of the A3-infected nymphs (4 out of 5) maintained B. burgdorferi through the molt, and spirochetes cultured from the A3-LA7TC outgrowths were fluorescent when stained with FlAsH dye (data not shown).

Lastly, transmission was assessed by allowing the remaining infected nymphs to feed on naïve mice. All naïve mice fed upon by infected nymphs acquired B. burgdorferi (5 out of 5), as verified by the presence of spirochetes in tissue outgrowths; when spirochetes in a subset of those outgrowths were subjected to FlAsH assays, A3-LA7TC spirochetes were fluorescent (Figure 5A top-right panel). In addition, although technically challenging due to the absolute number of spirochetes in fed nymph midguts, it was possible to detect fluorescent spirochetes directly from A3-LA7TC infected, engorged nymphs that had been mechanically-disrupted and FlAsH-labeled (Figure 5A, lower right panel). Replete ticks were collected and a subset (4 per strain) was used to estimate the number of spirochetes per tick. On average, there was no significant difference in spirochete burden between A3- and A3-LA7TC-infected ticks $\left(3.04 \times 10^{4}\right.$ vs. $3.48 \times 10^{4}$ spirochetes per tick, respectively; $p=0.89$; Figure 5B). Taken together, the overall infection and fluorescence data demonstrate that spirochetes bearing a tetracysteine motif 
A

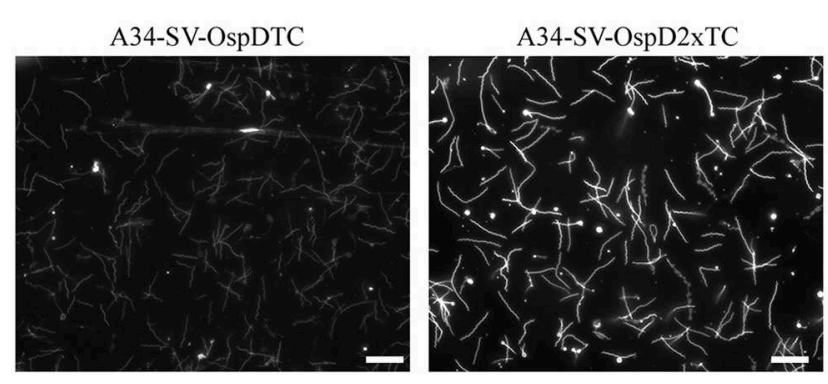

B31 A34 wild type
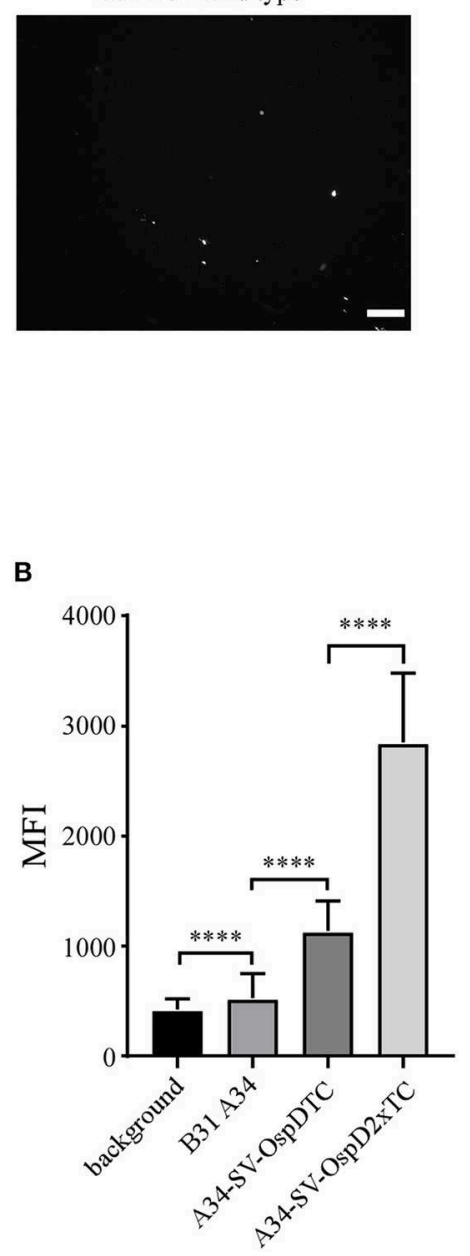

C

B31 A34
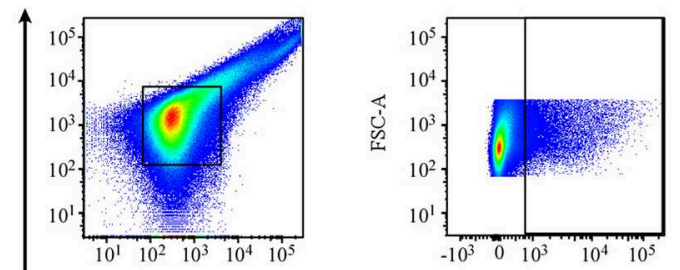

A34-SV-OspDTC

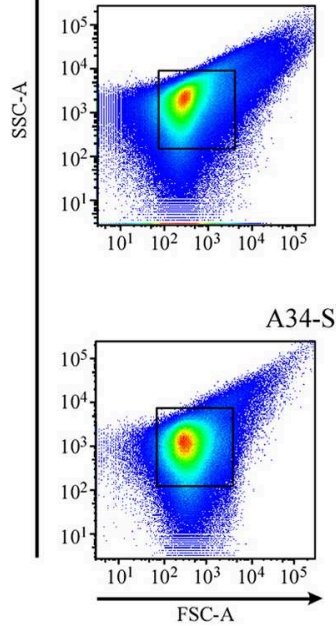

34-SV-OspD2xTC

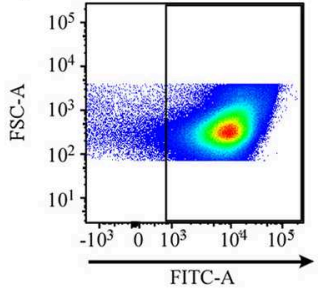

D

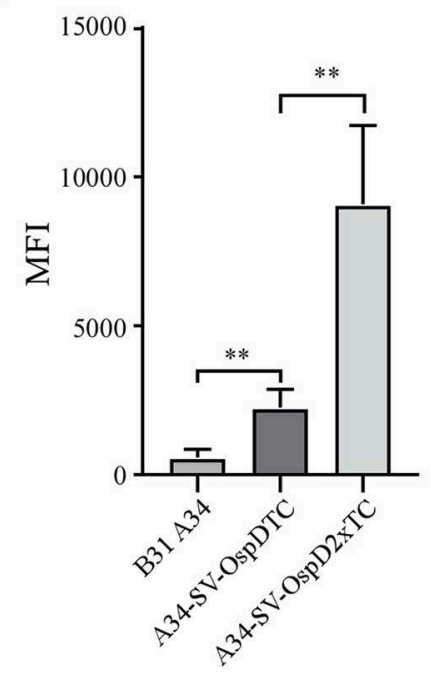

FIGURE 4 | Optimization and quantification of the FIAsH dye system for B. burgdorferi membrane proteins containing tetracysteine (TC) motifs. (A) Spirochetes bearing OspD with two tetracysteine motifs fluoresce more brightly than those carrying OspD with a single TC tag. Unmodified B. burgdorferi strain B31 A34 does not fluoresce in the presence of FIAsH dye, but addition of a single tetracysteine-tag to OspD (A34-SV-OspDTC) allows FIAsH binding and fluorescence. Addition of a second tetracysteine motif to OspD (A34-SV-OspD2xTC) further increases fluorescence. All images were captured with identical exposure times and not inverted. Scale bar $=10 \mu \mathrm{m}$. (B) Quantification of differences in relative fluorescence of $B$. burgdorferi strains by microscopy. Addition of a second tetracysteine motif on OspD significantly increased fluorescence intensity (A34-SV-OspDTC 1,136 $\pm 272.3 \mathrm{MFI}$ vs. A34-SV-OspD2xTC 2,850 \pm 628.5 MFI, Mann-Whitney test $p<0.0001$ ). Background in areas where there were no spirochetes, as well as of $B$. burgdorferi cells lacking tetracysteine motifs, were $421.1 \pm 95.95 \mathrm{MFI}$ and $524 \pm 225.6 \mathrm{MFI}$, respectively. Individual spirochete fluorescence was quantified using Nikon Elements software version 4.2 and compiled to generate an average fluorescence per strain. Mean Fluorescence Intensity $=$ MFI. Standard deviation is depicted by error bars $\left.{ }^{\star \star \star \star \star}\right) P<0.0001$. (C) Detection and differentiation of FIAsH-labeled spirochetes containing a single or double tetracysteine motif by flow cytometry. Addition of a second tetracysteine motif on OspD significantly increased fluorescence intensity (geometric mean for A34-SV-OspDTC 2,247 $\pm 603.5 \mathrm{MFI}$ vs. A34-SV-OspD2xTC 9,079 $\pm 2,648 \mathrm{MFI}, p<0.0079$ ). FSC-A, forward scatter; SSC-A, side-scatter; FITC-A, measure of fluorescence-intensity. (D) Quantification of the geometric mean data from 5 independent flow cytometer runs analyzed in Prism for statistical significance. Mean Fluorescence Intensity $=$ MFI. Standard deviation is depicted by the error bars $\left.{ }^{\star \star}\right) P<0.008$. 


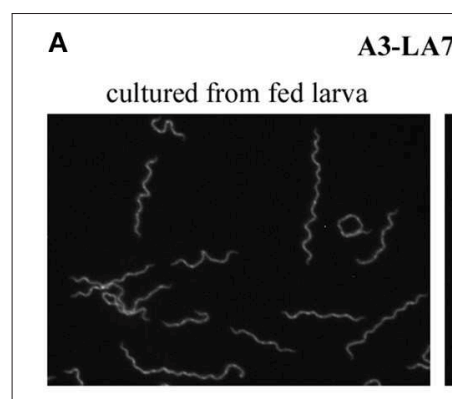

A3-LA7TC
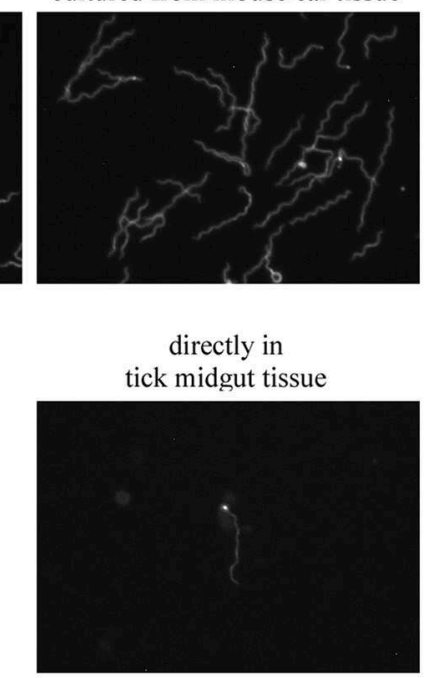

B

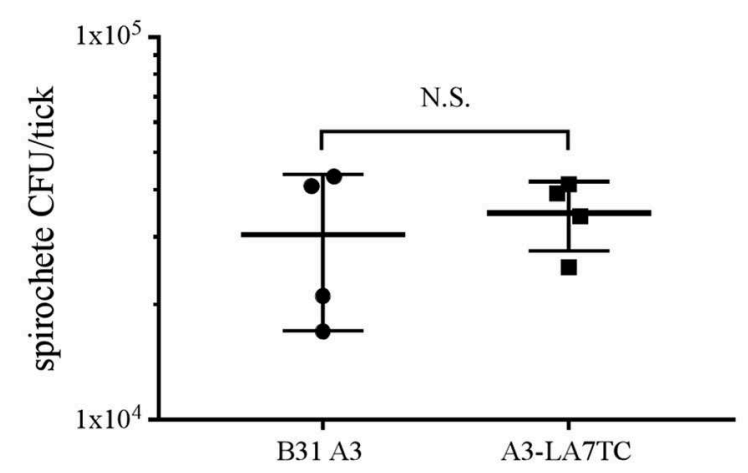

FIGURE $\mathbf{5} \mid$ B. burgdorferi strain with tetracysteine-tagged inner membrane protein $L A 7$ can colonize and be transmitted between the tick vector and murine host. (A) FIAsH-labeling of A3-LA7TC after isolation from tick larvae that fed on infected mice (left), and from ear tissue of mice fed on by infected nymphs (right). Spirochete labeled directly in midgut tissue dissected from an engorged nymph (lower panel). Images have been cropped and are representative of what was observed. (B) Strain with tetracysteine-tagged LA7 (A3-LA7TC) colonizes ticks as efficiently as WT (B31 A3). Spirochete burden was determined as CFU in fed infected nymphs by individually macerating and plating 4 replete ticks per group after feeding on naïve mice. The mean and standard deviation bars are shown. No significant difference was detected between strains using the Mann-Whitney test. Standard deviation is represented by the error bars (N.S. $=$ Not Significant) $P<0.89$.

on LA7 exhibit a WT phenotype throughout the mouse-tickmouse infectious cycle and can be FlAsH-labeled either directly in tick midgut tissue or after in vitro cultivation of spirochetes from tick or murine tissues.

\section{FIAsH-labeled Proteins Retain Fluorescence Over Time}

We utilized biarsenical dyes to assess the length of time FlAsHlabeled protein could be detected during in vitro cultivation.
As previously described, we complemented strain A3 $\Delta$ OspD in trans with a double tetracysteine-tagged copy of ospD on a shuttle vector $(\Delta \mathrm{OspD}-\mathrm{SV}-2 \mathrm{xTC})$. We used this complemented strain in time-course experiments in order to eliminate potential competition with WT OspD and have only the tetracysteinetagged OspD variant available for processing and insertion into the outer membrane. $\triangle$ OspD-SV-2xTC spirochetes were pelleted, labeled with FlAsH dye, and then washed and resuspended in BSK culture medium. When fluorescence was monitored during subsequent in vitro growth at $35^{\circ} \mathrm{C}$, the signal diminished almost entirely after $24 \mathrm{~h}$ (data not shown). Presumably this observed loss of fluorescence was due to dilution of FlAsH-labeled OspD by growth and cell division that occurred within this timeframe $(\sim 5$ doublings and $\sim 30$-fold increase in cell number). Therefore, we performed this experiment at $22^{\circ} \mathrm{C}$, a condition in which Borrelia grows more slowly, to reduce the dilution of labeled protein resulting from cell growth and division. Fluorescence of the $\triangle \mathrm{OspD}-\mathrm{SV}-2 \mathrm{xTC}$ strain was monitored over the course of 8 days (Figure 6A). Spirochetes of the same strain that were FlAsH-labeled and then heatkilled were included as a metabolically inert control that does not undergo cell division or have an active mechanism for removing or shedding protein (Figure 6B). $\triangle \mathrm{OspD}-\mathrm{SV}-2 \mathrm{xTC}$ fluorescence diminished over the time course, compared to day 0 , but remained detectable. Passive loss of fluorescence of the FlAsH signal did not appear to be an issue, as fluorescence was relatively stable throughout the time course for the heat-killed samples.

At each time point, we relabeled the cells with $\mathrm{ReAsH}$, a red variant of biarsenical dyes (Figure 6C). This pulse-chase style experiment allowed visualization of cells that bound both FlAsH and ReAsH over time. At day 0, very little ReAsH was taken up by cells, whereas the ReAsH signal grew appreciably by days 4 and 8 , These data suggest that binding sites for $\mathrm{ReAsH}$ became available as FlAsH-labeled OspDTC waned and unlabeled OspDTC increased in the spirochete membrane over time.

\section{Discussion}

In this study we describe the application of a new technique in spirochetes for fluorescently labeling specific proteins using tetracysteine tags and biarsenical dyes. This technique allowed fluorescent labeling of spirochetes by incorporating a 6 amino acid motif at the C-terminus of both inner or outer membrane proteins, and staining with a membrane-permeable biarsenical dye that binds to this motif. The small increase in protein size conferred by the tetracysteine motif minimizes potential negative effects that may occur when targets are fused to larger fluorogenic proteins (e.g., GFP or RFP), such as steric hindrance, causing improper protein folding, trafficking to the incorrect cellular location, or inappropriate oligomerization. We successfully applied the same protocol to the distantly related spirochetes $B$. burgdorferi, a zoonotic human pathogen, and $L$. biflexa, a free-living saprophyte, suggesting that the $\mathrm{FlAsH}$ system can be applied broadly across all spirochete species.

This technique worked well for both the outer membrane protein OspD and the inner membrane protein LA7 of $B$. burgdorferi, indicating that the dye is freely diffusible across 


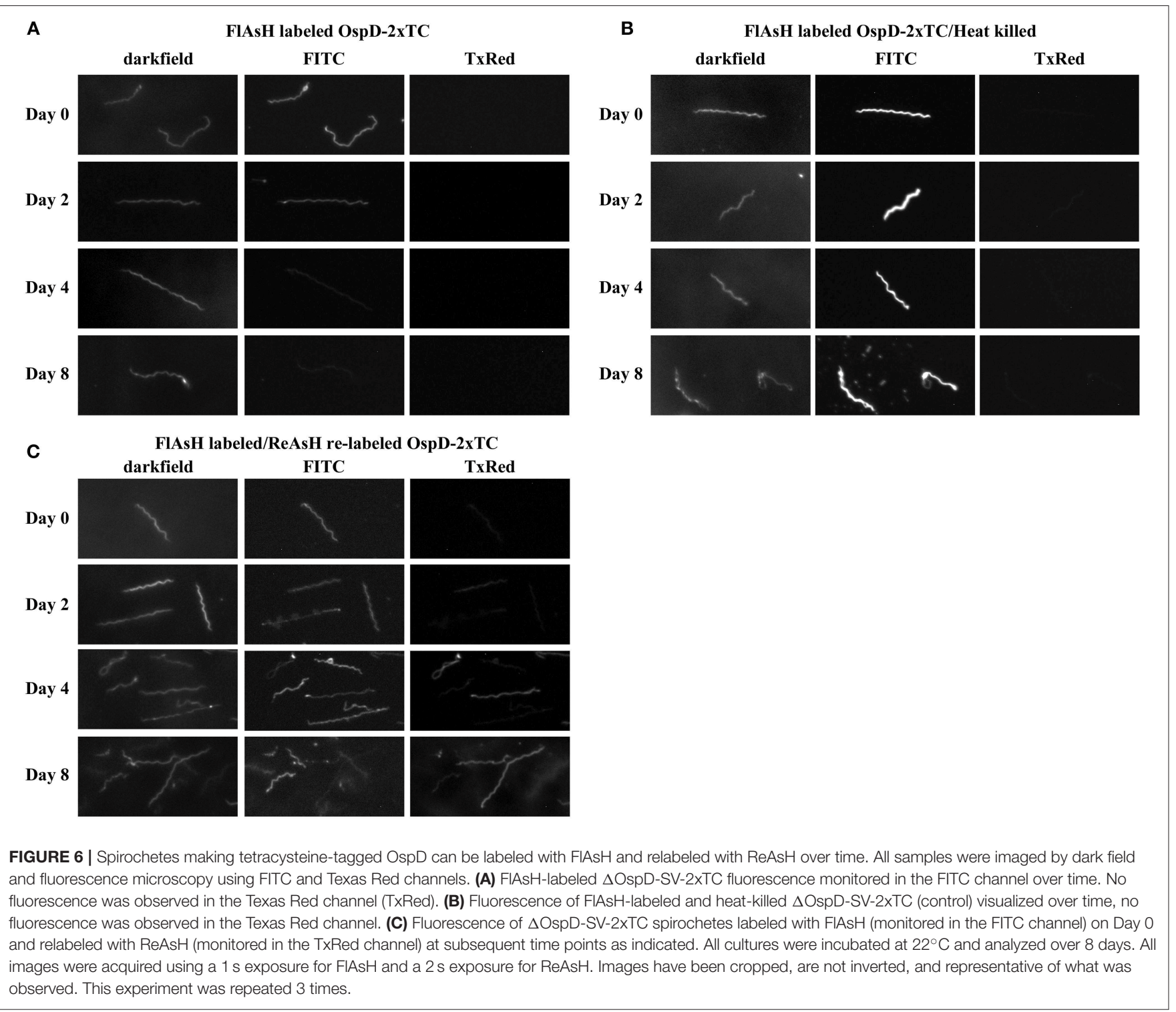

the intact outer membrane of living spirochetes without the need for fixation or permeabilization. Although the membrane location of OmpA in L. biflexa has not been definitively verified, it is predicted to possess a lipoprotein signal peptide by LipoP 1.0 (http://www.cbs.dtu.dk/services/LipoP/) and was identified in the membrane-associated protein fraction of leptospires (Stewart et al., 2016). Further, ompA-TC and la7-TC constructs were targeted to their respective endogenous loci, while the ospD-TC fusions were expressed in trans from shuttle vectors, indicating that single copy (endogenous locus) or multicopy (shuttle vector) expression sites are both effective options.

As with any technique, the FlAsH system has limitations and required some optimization. Biarsenical dyes rely on precisely spaced and oriented thiol groups to bind and fluoresce. However, due to the cellular abundance of protein thiols, non-specific binding can occur and occlude proper protein visualization. This can be remedied by reducing protein disulfide bonds in the cellular environment before labeling with dithiol compounds such as 1,4-dithiothreitol (DTT) or 2,3-dimercaptopropanol (BAL). Unexpectedly, we noticed that heat-killed, wild-type spirochetes will non-specifically bind the FlAsH dye. It does not appear to be general autofluorescence of dead spirochetes because the fluorescent signal is only detectable in the FITC channel, not the Texas Red, or DAPI channels, and requires the FlAsH dye. In an attempt to alleviate this issue, we increased the DTT levels 10-fold, which lowered fluorescence in both viable and dead spirochetes. We speculate that heat-killed spirochetes have exposed di-sulfide bonds that are otherwise inaccessible, resulting in the observed binding of the biarsenical dye.

Biarsenical dyes photo-bleach faster than fluorescent proteins, and the fluorescent signal of a tetracysteine-tagged protein is typically several fold lower than that of a cognate GFP fusion (Adams et al., 2002; Crivat et al., 2011). Also, proteins less abundant than OmpA, OspD, or LA7 have not been tested. 
However, these limitations could potentially be mitigated by adding a dual tetracysteine motif to the protein of interest, which not only increases signal intensity, but also prolongs fluorescence. HeLa cells transiently transfected with a tetracysteine motif on $\alpha$-tubulin indicated a positive linear relationship between additional tetracysteine tags and fluorescence intensity (Van Engelenburg et al., 2010). Our results with OspD-TC in B. burgdorferi agree with this finding.

We also noted loss of fluorescence within $24 \mathrm{~h}$ when FlAsHlabeled spirochetes were cultured at $35^{\circ} \mathrm{C}$, presumably due to cell growth and division, where segregation of labeled protein to daughter cells combined with synthesis of new protein leads to dilution of the signal on individual bacteria. We are not aware of similar studies examining the duration of fluorescent protein fusions in B. burgdorferi as a comparator. However, when grown at $22^{\circ} \mathrm{C}, \mathrm{B}$. burgdorferi replication is significantly slower and fluorescence could be monitored over a longer time frame. At this temperature, we were able to follow FlAsH dye-stained OspD on the surface of B. burgdorferi for 8 days, albeit with diminishing signal. Re-labeling assays (using the red-variant, $\mathrm{ReAsH}$ ) indicated that there was very little OspD available to relabel at the onset of the experiment, suggesting that the initial dye labeling was highly efficient. With time, OspD protein became available for re-labeling and we observed increased fluorescence with ReAsH. This is in contrast to spirochetes that were heatkilled and do not lose fluorescence over time. Together, this would suggest that the decrease in FlAsH fluorescence with time in culture is not due to diffusion of the dye into the media, but to cell division and new protein synthesis, leading to dilution of the labeled protein in the daughter cells. However, another possible explanation for loss of fluorescence could be due to shedding of labeled protein into the media via membrane bound vesicles, or active release by an undefined mechanism.

A significant attribute of the biarsenical dye system is the small size of the tetracysteine motif, which does not impede critical protein function in vivo. Previous work on LA7 indicated a role in tick acquisition, with upregulation of expression of the gene encoding LA7 (bb0365) in feeding ticks (Pal et al., 2008). LA7-deficient spirochetes were severely impaired in both tick acquisition and transmission. Here, we show that a strain with an engineered tetracysteine motif on LA7 is fully competent in an experimental mouse-tick-mouse infectious cycle. No difference in the spirochete burden per nymphal tick was observed between TC-tagged and WT strains. When subjected to FlAsH assays, TC-tagged spirochetes isolated from mouse tissues or ticks fluoresced, indicating retention of the tetracysteine motif on the protein. A further advantage of TC-tagged spirochetes is the ability to directly visualize live spirochetes in dissected tick tissues, allowing assessment of target gene expression without fixation or culturing. Together, these data demonstrate that the gene encoding a TC-tagged protein is stably maintained throughout the infectious cycle and that addition of the TC tag does not interfere with the function of a protein that is important for maintenance in the tick. An additional benefit of this system over other fluorogenic methods includes the freely diffusible nature of biarsenical dyes, allowing labeling of sub-surface proteins in live spirochetes without fixation or permeabilization.
We demonstrate that tetracysteine motifs coupled with biarsenical dyes are capable of labeling abundant membrane proteins in both B. burgdorferi and L. biflexa, and likely can be extended to proteins in other cellular locations in these and other spirochetes. Compared to their fluorescent protein counterparts, the small size of the tetracysteine motifs makes them ideal for protein localization and trafficking studies. Genetically encoded tetracysteine motifs coupled with biarsenical dyes represent a novel molecular tool for studying spirochetes, and can provide valuable insights into spirochete morphology, protein-protein interactions, and cellular trafficking.

\section{DATA AVAILABILITY}

All datasets generated for this study are included in the manuscript/Supplementary Files.

\section{AUTHOR CONTRIBUTIONS}

PS conceived and supervised the study with input from PR. CH, HS, MS, and TE contributed to the design and conducted the FlAsH dye experiments. VC and JW assisted with the animal study. AC analyzed samples by flow cytometry. TS provided advice and assistance with image analyses. $\mathrm{CH}$ took the lead role in manuscript preparation with direction and assistance from PS and PR.

\section{FUNDING}

This research was supported by the Intramural Research Program of the National Institute of Allergy and Infectious Diseases, National Institutes of Health, and salary support for MS by the Technology Agency of the Czech Republic (TE01020118).

\section{ACKNOWLEDGMENTS}

We would like to thank Dr. Gerry Baron for development of the FlAsH-dye protocol that we employed, Dr. Elsio Wunder for providing the vectors used for conjugation with Leptospira, and Dr. Brian Stevenson for the LA7 antibody. In addition, we appreciate the graphical expertise of Anita Mora, Ryan Kissinger, and Austin Athman in figure preparation. We thank Drs. Karin Aistleitner and Paul Beare for critical reading of the manuscript and insightful comments.

\section{SUPPLEMENTARY MATERIAL}

The Supplementary Material for this article can be found online at: https://www.frontiersin.org/articles/10.3389/fcimb. 2019.00287/full\#supplementary-material

Supplementary Figure $1 \mid \mathrm{OspD}$ and LA7 protein levels in B31 strains. (A) Immunoblot analysis using antisera recognizing OspD ( 28 kDa). OspD protein levels are comparable in strains transformed with SV-OspDTC and SV-OspD2xTC (B) Immunoblot analysis using antisera recognizing LA7 ( 22 kDa). LA7 protein level is comparable between strains B31 A3 and A3-LA7TC. (C,D) Coomassie brilliant blue-stained polyacrylamide gel indicating relative protein loads of samples used for immunoblots in $(\mathbf{A}, \mathbf{B})$, respectively. 
Supplementary Figure 2 | Duration of fluorescence increased by the addition of a second tetracysteine motif. Fluorescence of strain A34-SV-OspDTC was compared to A34-SV-OspD2xTC over time. Videos were taken with Nikon Elements software and data analyzed in Prism. Measurements were taken of three spirochetes/strain during the same experiment. Fluorescence represents the intensity of spirochetes measured with the Nikon Elements software; time measured in seconds (s).

Supplementary Figure $\mathbf{3}$ | Non-specific binding of FIAsH dye to heat-killed spirochetes. A3 spirochetes that do not contain a tetracysteine motif, but were heat killed prior to FIAsH staining, readily take up the dye and fluoresce (1st row)

\section{REFERENCES}

Adams, S. R., Campbell, R. E., Gross, L. A., Martin, B. R., Walkup, G. K., Yao, Y., et al. (2002). New biarsenical ligands and tetracysteine motifs for protein labeling in vitro and in vivo: synthesis and biological applications. J. Am. Chem. Soc. 124, 6063-6076. doi: 10.1021/ja017687n

Andresen, M., Schmitz-Salue, R., and Jakobs, S. (2004). Short tetracysteine tags to beta-tubulin demonstrate the significance of small labels for live cell imaging. Mol. Biol. Cell 15, 5616-5622. doi: 10.1091/mbc.e04-06-0454

Arhel, N., Genovesio, A., Kim, K. A., Miko, S., Perret, E., Olivo-Marin, J. C., et al. (2006). Quantitative four-dimensional tracking of cytoplasmic and nuclear HIV-1 complexes. Nat. Methods 3, 817-824. doi: 10.1038/nmeth928

Aviat, F., Slamti, L., Cerqueira, G. M., Lourdault, K., and Picardeau, M. (2010). Expanding the genetic toolbox for Leptospira species by generation of fluorescent bacteria. Appl. Environ. Microbiol. 76, 8135-8142. doi: 10.1128/AEM.02199-10

Bockenstedt, L. K., Gonzalez, D. G., Haberman, A. M., and Belperron, A. A. (2012). Spirochete antigens persist near cartilage after murine Lyme borreliosis therapy. J. Clin. Invest. 122, 2652-2660. doi: 10.1172/JCI58813

Burgdorfer, W., Barbour, A. G., Hayes, S. F., Benach, J. L., Grunwaldt, E., and Davis, J. P. (1982). Lyme disease-a tick-borne spirochetosis? Science 216, 1317-1319.

Bykowski, T., Babb, K., von Lackum, K., Riley, S. P., Norris, S. J., and Stevenson, B. (2006). Transcriptional regulation of the Borrelia burgdorferi antigenically variable VlsE surface protein. J. Bacteriol. 188, 4879-4889. doi: 10.1128/JB.00229-06

Carrasco, S. E., Troxell, B., Yang, Y., Brandt, S. L., Li, H., Sandusky, G. E., et al. (2015). Outer surface protein OspC is an antiphagocytic factor that protects Borrelia burgdorferi from phagocytosis by macrophages. Infect. Immun. 83, 4848-4860. doi: 10.1128/IAI.01215-15

Carroll, J. A., Stewart, P. E., Rosa, P., Elias, A. F., and Garon, C. F. (2003). An enhanced GFP reporter system to monitor gene expression in Borrelia burgdorferi. Microbiology 149(Pt 7), 1819-1828. doi: 10.1099/mic.0.26165-0

Cerqueira, G. M., Souza, N. M., Araujo, E. R., Barros, A. T., Morais, Z. M., Vasconcellos, S. A., et al. (2011). Development of transcriptional fusions to assess Leptospira interrogans promoter activity. PLOS ONE 6:e17409. doi: 10.1371/journal.pone.0017409

Clifton, D. R., Nolder, C. L., Hughes, J. L., Nowalk, A. J., and Carroll, J. A. (2006). Regulation and expression of bba66 encoding an immunogenic infectionassociated lipoprotein in Borrelia burgdorferi. Mol. Microbiol. 61, 243-258. doi: 10.1111/j.1365-2958.2006.05224.x

Copeland, M. F., Flickinger, S. T., Tuson, H. H., and Weibel, D. B. (2010). Studying the dynamics of flagella in multicellular communities of Escherichia coli by using biarsenical dyes. Appl. Environ. Microbiol. 76, 1241-1250. doi: 10.1128/AEM.02153-09

Crivat, G., Tokumasu, F., Sa, J. M., Hwang, J., and Wellems, T. E. (2011). Tetracysteine-based fluorescent tags to study protein localization and trafficking in Plasmodium falciparum-infected erythrocytes. PLOS ONE 6:e22975. doi: 10.1371/journal.pone.0022975

Dunham-Ems, S. M., Caimano, M. J., Pal, U., Wolgemuth, C. W., Eggers, C. H., Balic, A., et al. (2009). Live imaging reveals a biphasic mode of dissemination of Borrelia burgdorferi within ticks. J. Clin. Invest. 119, 3652-3665. doi: 10.1172/JCI39401

Eggers, C. H., Caimano, M. J., and Radolf, J. D. (2006). Sigma factor selectivity in Borrelia burgdorferi: RpoS recognition of the ospE/ospF/elp promoters is similarly to A3-LA7TC spirochetes containing a tetracysteine motif (2nd row). Spirochetes that have been heat-killed but not incubated with FIAsH, do not fluoresce (3rd and 4th row). Fluorescent spirochetes are only detected in the FITC channel, not in any other channels (not shown).

Supplementary Figure $\mathbf{4} \mid$ B. burgdorferi wild-type spirochetes (B31 A3) isolated from tick vectors and murine hosts do not fluoresce after labeling. FIAsH-labeling of spirochetes isolated from larval ticks fed on B31 A3-infected mice (top); spirochetes isolated from ear tissue of mice fed on by B31-A3 infected nymphs (middle); and spirochete in dissected midgut of an engorged B31 A3-infected nymph (bottom). Representative dark field and FITC images are shown.

dependent on the sequence of the -10 region. Mol. Microbiol. 59, 1859-1875. doi: 10.1111/j.1365-2958.2006.05066.x

Elias, A. F., Bono, J. L., Kupko, J. J. III., Stewart, P. E., Krum, J. G., and Rosa, P. A. (2003). New antibiotic resistance cassettes suitable for genetic studies in Borrelia burgdorferi. J. Mol. Microbiol. Biotechnol. 6, 29-40. doi: $10.1159 / 000073406$

Elias, A. F., Stewart, P. E., Grimm, D., Caimano, M. J., Eggers, C. H., Tilly, K., et al. (2002). Clonal polymorphism of Borrelia burgdorferi strain B31 MI: implications for mutagenesis in an infectious strain background. Infect. Immun. 70, 2139-2150. doi: 10.1128/iai.70.4.2139-2150.2002

Enninga, J., Mounier, J., Sansonetti, P., and Tran Van Nhieu, G. (2005). Secretion of type III effectors into host cells in real time. Nat. Methods 2, 959-965. doi: 10.1038/nmeth804

Frank, K. L., Bundle, S. F., Kresge, M. E., Eggers, C. H., and Samuels, D. S. (2003). aadA confers streptomycin resistance in Borrelia burgdorferi. J. Bacteriol. 185, 6723-6727. doi: 10.1128/jb.185.22.6723-6727.2003

Gaspersic, J., Hafner-Bratkovic, I., Stephan, M., Veranic, P., Bencina, M., Vorberg, I., et al. (2010). Tetracysteine-tagged prion protein allows discrimination between the native and converted forms. FEBS J. 277, 2038-2050. doi: 10.1111/j.1742-4658.2010.07619.x

Gautam, A., Hathaway, M., McClain, N., Ramesh, G., and Ramamoorthy, R. (2008). Analysis of the determinants of bba64 (P35) gene expression in Borrelia burgdorferi using a gfp reporter. Microbiology 154(Pt 1), 275-285. doi: 10.1099/mic.0.2007/011676-0

Gautam, A., Hathaway, M., and Ramamoorthy, R. (2009). The Borrelia burgdorferi flaB promoter has an extended -10 element and includes a T-rich $-35 /-10$ spacer sequence that is essential for optimal activity. FEMS Microbiol. Lett. 293, 278-284. doi: 10.1111/j.1574-6968.2009.01542.x

Grewe, C., and Nuske, J. H. (1996). Immunolocalization of a $22 \mathrm{kDa}$ protein (IPLA7, P22) of Borrelia burgdorferi. FEMS Microbiol. Lett. 138, 215-219. doi: 10.1111/j.1574-6968.1996.tb08160.x

Griffin, B. A., Adams, S. R., and Tsien, R. Y. (1998). Specific covalent labeling of recombinant protein molecules inside live cells. Science 281, 269-272.

Grove, A. P., Liveris, D., Iyer, R., Petzke, M., Rudman, J., Caimano, M. J., et al. (2017). Two distinct mechanisms govern RpoS-mediated repression of tick-phase genes during mammalian host adaptation by Borrelia burgdorferi, the Lyme Disease Spirochete. MBio 8:4. doi: 10.1128/mBio. 01204-17

Jewett, M. W., Byram, R., Bestor, A., Tilly, K., Lawrence, K., Burtnick, M. N., et al. (2007). Genetic basis for retention of a critical virulence plasmid of Borrelia burgdorferi. Mol. Microbiol. 66, 975-990. doi: 10.1111/j.1365-2958.2007.05969.x

Krishnavajhala, A., Wilder, H. K., Boyle, W. K., Damania, A., Thornton, J. A., Perez de Leon, A. A., et al. (2017). Imaging of Borrelia turicatae producing the green fluorescent protein reveals persistent colonization of the Ornithodoros turicata midgut and salivary glands from nymphal acquisition through transmission. Appl. Environ. Microbiol. 83:5. doi: 10.1128/AEM.02503-16

Louvel, H., and Picardeau, M. (2007). Genetic manipulation of Leptospira biflexa. Curr. Protoc. Microbiol. Chapter 12, Unit 12E 14. doi: 10.1002/9780471729259.mc12e04s05

Matsunaga, J., and Haake, D. A. (2018). Identification of a cis-acting determinant limiting expression of sphingomyelinase gene sph2 in Leptospira interrogans with a gfp reporter plasmid. Appl. Environ. Microbiol. 84:e02068-18. doi: 10.1128/AEM.02068-18 
Miller, J. C., von Lackum, K., Woodman, M. E., and Stevenson, B. (2006). Detection of Borrelia burgdorferi gene expression during mammalian infection using transcriptional fusions that produce green fluorescent protein. Microb. Pathog. 41, 43-47. doi: 10.1016/j.micpath.2006.04.004

Moriarty, T. J., Norman, M. U., Colarusso, P., Bankhead, T., Kubes, P., and Chaconas, G. (2008). Real-time high resolution 3D imaging of the Lyme disease spirochete adhering to and escaping from the vasculature of a living host. PLoS Pathog. 4:e1000090. doi: 10.1371/journal.ppat.100 0090

Norman, M. U., Moriarty, T. J., Dresser, A. R., Millen, B., Kubes, P., and Chaconas, G. (2008). Molecular mechanisms involved in vascular interactions of the Lyme disease pathogen in a living host. PLoS Pathog 4:e1000169. doi: 10.1371/journal.ppat.1000169

Pal, U., Dai, J., Li, X., Neelakanta, G., Luo, P., Kumar, M., et al. (2008). A differential role for BB0365 in the persistence of Borrelia burgdorferi in mice and ticks. J. Infect. Dis. 197, 148-155. doi: 10.1086/ 523764

Panchal, R. G., Ruthel, G., Kenny, T. A., Kallstrom, G. H., Lane, D., Badie, S. S., et al. (2003). In vivo oligomerization and raft localization of Ebola virus protein VP40 during vesicular budding. Proc. Natl. Acad. Sci. U.S.A. 100, 15936-15941. doi: 10.1073/pnas.2533915100.

Samuels, D. S., Mach, K. E., and Garon, C. F. (1994). Genetic transformation of the Lyme disease agent Borrelia burgdorferi with coumarin-resistant gyrB. J. Bacteriol. 176, 6045-6049.

Schulze, R. J., Chen, S., Kumru, O. S., and Zuckert, W. R. (2010). Translocation of Borrelia burgdorferi surface lipoprotein OspA through the outer membrane requires an unfolded conformation and can initiate at the C-terminus. Mol. Microbiol. 76, 1266-1278. doi: 10.1111/j.1365-2958.2010. 07172.x

Schulze, R. J., and Zuckert, W. R. (2006). Borrelia burgdorferi lipoproteins are secreted to the outer surface by default. Mol Microbiol 59, 1473-1484. doi: 10.1111/j.1365-2958.2006.05039.x

Senf, F., Tommassen, J., and Koster, M. (2008). Polar secretion of proteins via the Xcp type II secretion system in Pseudomonas aeruginosa. Microbiology 154(Pt. 10), 3025-3032. doi: 10.1099/mic.0.2008/018069-0

Stewart, P. E., Bestor, A., Cullen, J. N., and Rosa, P. A. (2008). A tightly regulated surface protein of Borrelia burgdorferi is not essential to the mouse-tick infectious cycle. Infect. Immun. 76, 1970-1978. doi: 10.1128/IAI.00714-07

Stewart, P. E., Carroll, J. A., Olano, L. R., Sturdevant, D. E., and Rosa, P. A. (2016). Multiple posttranslational modifications of Leptospira biflexa proteins as revealed by proteomic analysis. Appl. Environ. Microbiol. 82, 1183-1195. doi: 10.1128/AEM.03056-15

Takacs, C. N., Kloos, Z. A., Scott, M., Rosa, P. A., and Jacobs-Wagner, C. (2018). Characterization of fluorescent proteins, promoters, and selectable markers for applications in the Lyme disease spirochete Borrelia burgdorferi. Appl Environ Microbiol. 84:24. doi: 10.1128/AEM.01824-18
Teixeira, R. C., Baeta, B. A., Ferreira, J. S., Medeiros, R. C., Maya-Monteiro, C. M., Lara, F. A., et al. (2016). Fluorescent membrane markers elucidate the association of Borrelia burgdorferi with tick cell lines. Braz. J. Med. Biol. Res. 49:7. doi: 10.1590/1414-431X20165211

Van Engelenburg, S. B., Nahreini, T., and Palmer, A. E. (2010). FACS-based selection of tandem tetracysteine peptides with improved ReAsH brightness in live cells. Chembiochem 11, 489-493. doi: 10.1002/cbic.200900689

von Lackum, K., Ollison, K. M., Bykowski, T., Nowalk, A. J., Hughes, J. L., Carroll, J. A., et al. (2007). Regulated synthesis of the Borrelia burgdorferi inner-membrane lipoprotein IpLA7 (P22, P22-A) during the Lyme disease spirochaete's mammal-tick infectious cycle. Microbiology 153(Pt 5), 1361-1371. doi: 10.1099/mic.0.2006/003350-0

Whetstine, C. R., Slusser, J. G., and Zuckert, W. R. (2009). Development of a single-plasmid-based regulatable gene expression system for Borrelia burgdorferi. Appl. Environ. Microbiol. 75, 6553-6558. doi: 10.1128/AEM. 02825-08

Xu, H., Raddi, G., Liu, J., Charon, N. W., and Li, C. (2011). Chemoreceptors and flagellar motors are subterminally located in close proximity at the two cell poles in spirochetes. J. Bacteriol. 193, 2652-2656. doi: 10.1128/JB. 01530-10

Yang, X., Hegde, S., Shroder, D. Y., Smith, A. A., Promnares, K., Neelakanta, G., et al. (2013). The lipoprotein La7 contributes to Borrelia burgdorferi persistence in ticks and their transmission to naive hosts. Microbes Infect. 15, 729-737. doi: 10.1016/j.micinf.2013.06.001

Zhang, K., Liu, J., Charon, N. W., and Li, C. (2015). Hypothetical protein BB0569 is essential for chemotaxis of the Lyme disease spirochete Borrelia burgdorferi. J. Bacteriol. 198, 664-672. doi: 10.1128/JB.00877-15

Zhao, Z., Zhao, Y., Zhuang, X. Y., Lo, W. C., Baker, M. A. B., Lo, C. J., et al. (2018). Frequent pauses in Escherichia coli flagella elongation revealed by single cell real-time fluorescence imaging. Nat. Commun. 9:1885. doi: 10.1038/s41467-018-04288-4

Conflict of Interest Statement: The authors declare that the research was conducted in the absence of any commercial or financial relationships that could be construed as a potential conflict of interest.

This work is authored by Hillman, Stewart, Strnad, Stone, Starr, Carmody, Evans, Carracoi, Wachter and Rosa on behalf of the U.S. Government and, as regards Hillman, Stewart, Strnad, Stone, Starr, Carmody, Evans, Carracoi, Wachter, Rosa, and the U.S. Government, is not subject to copyright protection in the United States. Foreign and other copyrights may apply. This is an open-access article distributed under the terms of the Creative Commons Attribution License (CC BY). The use, distribution or reproduction in other forums is permitted, provided the original author(s) and the copyright owner(s) are credited and that the original publication in this journal is cited, in accordance with accepted academic practice. No use, distribution or reproduction is permitted which does not comply with these terms. 